\title{
The Lusternik-Schnirelmann category and the fundamental group
}

\author{
Alexander DRANishniKOV
}

We prove that

$$
\operatorname{cat}_{\mathrm{LS}} X \leq \operatorname{cd}\left(\pi_{1}(X)\right)+\left\lceil\frac{\operatorname{dim} X-1}{2}\right\rceil
$$

for every CW-complex $X$ where $\operatorname{cd}\left(\pi_{1}(X)\right)$ denotes the cohomological dimension of the fundamental group of $X$.

$55 \mathrm{M} 30$

\section{Introduction}

The Lusternik-Schnirelmann category cat ${ }_{\mathrm{LS}} X$ of a topological space $X$ is the minimal number $n$ such that there is an open cover $\left\{U_{0}, \ldots, U_{n}\right\}$ of $X$ by $n+1$ contractible in $X$ sets (we note that sets $U_{i}$ are not necessarily contractible). The Lusternik-Schnirelmann category has proven useful in different areas of mathematics. In particular, the classical theorem of Lusternik and Schnirelmann (see Cornea et al [3]) proven in the 30s states that cat $_{\mathrm{LS}} M$ gives a lower bound for the number of critical points on $M$ of any smooth not necessarily Morse function. For nice spaces, such as CW-complexes, it is an easy observation that cat LS $X \leq \operatorname{dim} X$. In the 40s Grossman [8] (and independently in the 50s GW Whitehead $[16 ; 3]$ ) proved that for simply connected CW-complexes cat $_{\mathrm{LS}} X \leq \operatorname{dim} X / 2$. In the presence of a fundamental group as small as $\mathbb{Z}_{2}$ the Lusternik-Schnirelmann category can be equal to the dimension. An example is $\mathbb{R} P^{n}$.

Nevertheless, Yu Rudyak conjectured that in the case of free fundamental group there should be a Grossman-Whitehead-type inequality at least for closed manifolds. There were partial results towards Rudyak's conjecture (see Dranishnikov, Katz and Rudyak [6] and Strom [13]) until it was settled by the author [5]. Also it was shown in [5] that a Grossman-Whitehead-type estimate holds for complexes with fundamental group of cohomological dimension $\leq 2$. We recall that free groups (and only them by Stallings [12] and Swan [15]) have cohomological dimension one. In this paper we prove an inequality for complexes with fundamental groups having finite cohomological dimension. Complexes of type $\mathbb{C} P^{n} \times B \pi$ show that our inequality is sharp when $\pi$ is free. 
We conclude the introductory part with definitions and statements from [5] which are used in this paper. Let $\mathcal{U}=\left\{U_{\alpha}\right\}_{\alpha \in A}$ be a family of sets in a topological space $X$. Formally, it is a function $U: A \rightarrow 2^{X} \backslash\{\varnothing\}$ from the index set to the set of nonempty subsets of $X$. The sets $U_{\alpha}$ in the family $\mathcal{U}$ will be called elements of $\mathcal{U}$. The multiplicity of $\mathcal{U}$ (or the order) at a point $x \in X$, denoted $\operatorname{Ord}_{x} \mathcal{U}$, is the number of elements of $\mathcal{U}$ that contain $x$. The multiplicity of $\mathcal{U}$ is defined as $\operatorname{Ord} \mathcal{U}=\sup _{x \in X} \operatorname{Ord}_{x} \mathcal{U}$. A family $\mathcal{U}$ is a cover of $X$ if $\operatorname{Ord}_{x} \mathcal{U} \neq 0$ for all $x$. A cover $\mathcal{U}$ is a refinement of another cover $\mathcal{C}(\mathcal{U}$ refines $\mathcal{C})$ if for every $U \in \mathcal{U}$ there exists $C \in \mathcal{C}$ such that $U \subset C$. We recall that the covering dimension of a topological space $X$ does not exceed $n, \operatorname{dim} X \leq n$, if for every open cover $\mathcal{C}$ of $X$ there is an open refinement $\mathcal{U}$ with $\operatorname{Ord} \mathcal{U} \leq n+1$.

Definition 1.1 A family $\mathcal{U}$ of subsets of $X$ is called a $k$-cover, $k \in N$, if every subfamily of $k$ elements forms a cover of $X$.

The following is obvious (see Dranishnikov [5]).

Proposition 1.2 A family $\mathcal{U}$ that consists of $m$ subsets of $X$ is an $(n+1)$-cover of $X$ if and only if $\operatorname{Ord}_{x} \mathcal{U} \geq m-n$ for all $x \in X$.

The following theorem can be found in Ostrand [10].

Theorem 1.3 (Kolmogorov-Ostrand) A metric space $X$ is of dimension $\leq n$ if and only if for each open cover $\mathcal{C}$ of $X$ and each integer $m \geq n$, there exist $m$ disjoint families of open sets $\mathcal{V}_{0}, \ldots, \mathcal{V}_{m}$ such that their unions $\bigcup \mathcal{V}_{i}$ is an $(n+1)$-cover of $X$ and it refines $\mathcal{C}$.

Let $f: X \rightarrow Y$ be a map and let $X^{\prime} \subset X$. A set $U \subset X$ is fiberwise contractible to $X^{\prime}$ if there is a homotopy $H: U \times[0,1] \rightarrow X$ such that $H(x, 0)=x, H(U \times\{1\}) \subset X^{\prime}$, and $f(H(x, t))=f(x)$ for all $x \in U$.

We refer to [5] for the proof of the following:

Theorem 1.4 Let $\mathcal{U}=\left\{U_{0}, \ldots, U_{k}\right\}$ be an open cover of a normal topological space $X$. Then for any $m=0,1,2, \ldots, \infty$ there is an open $(k+1)$-cover $\mathcal{U}_{m}=$ $\left\{U_{0}, \ldots, U_{k+m}\right\}$ of $X$ extending $\mathcal{U}$ such that for $n>k, U_{n}=\bigcup_{i=0}^{k} V_{i}$ is a disjoint union with $V_{i} \subset U_{i}$.

Corollary 1.5 Let $f: X \rightarrow Y$ be a continuous map of a normal topological space and let $\mathcal{U}=\left\{U_{0}, \ldots, U_{k}\right\}$ be an open cover of $X$ by sets fiberwise contractible to $X^{\prime} \subset X$. Then for any $m=0,1,2, \ldots, \infty$ there is an open $(k+1)$-cover $\mathcal{U}_{m}=\left\{U_{0}, \ldots, U_{k+m}\right\}$ of $X$ by sets fiberwise contractible to $X^{\prime}$. 


\section{Generalization of Ganea's fibrations}

Let $A \subset Z$ be a closed subset of a path-connected space and let $F$ denote the homotopy fiber of the inclusion. By $A_{Z}$ we denote the space of paths in $Z$ issued from $A$, ie the space of continuous maps $\phi:[0,1] \rightarrow Z$ with $\phi(0) \in A$ and we define a map $p_{A}: A_{Z} \rightarrow Z$ by the formula $p(\phi)=\phi(1)$. Note that $A_{Z}$ deforms to $A$ and $p_{A}$ is a Hurewicz fibration. Then by the definition $F$ is the fiber of $p_{A}$.

Proposition 2.1 There is a Hurewicz fibration $\pi: F \rightarrow A$ with fiber $\Omega Z$, the loop space on $Z$.

Proof The map $q^{\prime}: A_{Z} \rightarrow A \times Z$ that sends a path to the end points is a Hurewicz fibration as a pullback of the Hurewicz fibration $q: Z^{[0,1]} \rightarrow Z \times Z$ [11]. The fiber of $q$ is the loop space $\Omega Z$. Since $p_{A}=\operatorname{pr}_{2} \circ q^{\prime}$, the fiber $F=p_{A}^{-1}(x)=\left(q^{\prime}\right)^{-1} \operatorname{pr}_{2}^{-1}(x)=$ $q^{-1}(A)$ is the total space of a Hurewicz fibration $q$ over $A$ with the fiber $\Omega Z$.

We define the $k$-th generalized Ganea's fibration $p_{k}: E_{k}(Z, A) \rightarrow Z$ over a path connected space $Z$ with a fixed closed subset $A$ as the fiberwise join product of $k+1$ copies of the fibrations $p_{A}: A_{Z} \rightarrow Z$. Since $p_{A}$ is a Hurewicz fibration and the fiberwise join of Hurewicz fibrations is a Hurewicz fibration, so are all $p_{k}$ by Švarc [14]. Note that the fiber of $p_{k}$ is the join product $*^{k+1} F$ of $k+1$ copies of $F$ (see Cornea et al [3] for more details). Also we note that for $A=\left\{z_{0}\right\}$ the fibration $p_{k}$ is the standard Ganea fibration. The following is a generalization of the Ganea-Švarc theorem.

Theorem 2.2 Let $A \subset X$ be a subcomplex contractible in $X$. Then $\operatorname{cat}_{\mathrm{LS}}(X) \leq k$ if and only if the generalized Ganea fibration

$$
p_{k}: E_{k}(Z, A) \rightarrow Z
$$

admits a section.

Proof When $A$ is a point this statements turns into the classical Ganea-Švarc theorem [3; 14]. Since for $z_{0} \in A$, the above fibration $p_{k}: E_{k}\left(Z, z_{0}\right) \rightarrow Z$ is contained in $p_{k}: E_{k}(Z, A) \rightarrow Z$, the classical Ganea-Švarc theorem implies the only if direction.

The barycentric coordinates of a section to $p_{k}$ define an open cover $U_{0}, \ldots U_{k}$ of $U_{i}$ with each $U_{i}$ contractible to $A$. Since $A$ is contractible in $Z$, all sets $U_{i}$ are contractible in $Z$. 
We call a map $f: X \rightarrow Y$ a stratified locally trivial bundle (with two strata) with fiber $(Z, A)$ if there $X^{\prime} \subset X$, such that $\left(f^{-1}(y), g^{-1}(y)\right) \cong(Z, A)$ for all $y \in Y$, where $g=\left.f\right|_{X^{\prime}}$, and there is an open cover $\mathcal{U}=\{U\}$ of $Y$ such that $\left(f^{-1}(U), g^{-1}(U)\right)$ is homeomorphic as a pair to $(Z \times U, A \times U)$ by means of a fiber preserving homeomorphism. Such a bundle is called a trivial stratified bundle if one cant take $\mathcal{U}$ consisting of one element $U=Y$.

Now let $f: X \rightarrow Y$ be a stratified locally trivial bundle with a subbundle $g: X^{\prime} \rightarrow Y$ and a fiber $(Z, A)$. We define a space

$$
E_{0}=\left\{\phi \in C(I, X) \mid f \phi(I)=f \phi(0), \phi(0) \in g^{-1}(f \phi(0))\right\}
$$

to be the space of all paths $\phi$ in $f^{-1}(y)$ for all $y \in Y$ with the initial point in $g^{-1}(y)$. The topology in $E_{0}$ is inherited from $C(I, X)$. We define a map $\xi_{0}: E_{0} \rightarrow X$ by the formula $\xi_{0}(\phi)=\phi(1)$. Then $\xi_{k}: E_{k} \rightarrow X$ is defined as the fiberwise join of $k+1$ copies of $\xi_{0}$. Formally, we define inductively $E_{k}$ as a subspace of the join $E_{0} * E_{k-1}$ :

$$
E_{k}=\bigcup\left\{\phi * \psi \in E_{0} * E_{k-1} \mid \xi_{0}(\phi)=\xi_{k-1}(\psi)\right\},
$$

which is the union of all intervals $[\phi, \psi]=\phi * \psi$ with the endpoints $\phi \in E_{0}$ and $\psi \in E_{k-1}$ such that $\xi_{0}(\phi)=\xi_{k-1}(\psi)$. There is a natural projection $\xi_{k}: E_{k} \rightarrow X$ that takes all points of each interval $[\phi, \psi]$ to $\phi(0)$.

Note that when $f: X=Z \times Y \rightarrow Y$ is a trivial stratified bundle with the subbundle $g: A \times Y \rightarrow Y, A \subset Z$, then $E_{k}=E_{k}(Z, A) \times Y$ and $\xi_{k}=p_{k} \times 1_{Y}$ where $p_{k}:\left(E_{k}, A\right) \rightarrow Z$ is the generalized Ganea fibration.

Lemma 2.3 Let $f: X \rightarrow Y$ be a stratified locally trivial bundle between paracompact spaces with a fiber $(Z, A)$ in which $A$ is contractible in $Z$. Then:

(i) For each $k$ the map $\xi_{k}: E_{k} \rightarrow X$ is a Hurewicz fibration.

(ii) The fiber of $\xi_{k}$ is the join of $k+1$ copies of the fiber $F$ of $p_{A}: A_{Z} \rightarrow Z$.

(iii) If the projection $\xi_{k}$ has a section, then $X$ has an open cover $\mathcal{U}=\left\{U_{0}, \ldots, U_{k}\right\}$ by sets each of which admits a fiberwise deformation into $X^{\prime}$ where $g: X^{\prime} \rightarrow Y$ is the subbundle.

Proof (i) First, we note that this statement holds true for trivial stratified bundles. By the assumption there is a cover $\mathcal{U}$ of $Y$ such that $\left.f\right|_{f^{-1} U}: f^{-1}(U) \rightarrow U$ is a trivial stratified bundle and hence $\xi_{k}$ is a Hurewicz fibration over $f^{-1}(U)$ for all $U \in \mathcal{U}$. Then by Hurewicz [9] (see also Dold [4]) we conclude that $\xi_{k}$ is a Hurewicz fibration over $X$. 
(ii) We note that $\xi_{k}$ over $f^{-1}(y)$ coincides with the generalized Ganea fibration $p_{k}$ for $(Z, A)$. Therefore, the fiber of $\xi_{k}$ coincides with the fiber of $p_{k}$. Then we apply Proposition 2.1

(iii) Suppose $\xi_{k}$ has a section $\sigma: X \rightarrow E_{k}$. For each $x \in X$ the element $\sigma(x)$ of $*^{k+1} \Omega F$ can be presented as the $(k+1)$-tuple

$$
\sigma(x)=\left(\left(\phi_{0}, t_{0}\right), \ldots,\left(\phi_{k}, t_{k}\right)\right) \text { where } \sum t_{i}=1 \text { and } t_{i} \geq 0 .
$$

Here we use the notation $t_{i}=t_{i}(x)$ and $\phi_{i}=\phi_{i}^{x}$. Clearly, $t_{i}(x)$ and $\phi_{i}^{x}$ are continuous functions of $x$.

A section $\sigma: X \rightarrow E_{k}$ defines a cover $\mathcal{U}=\left\{U_{0}, \ldots, U_{k}\right\}$ of $X$ as follows:

$$
U_{i}=\left\{x \in X \mid t_{i}(x)>0\right\} .
$$

By the construction of $U_{i}$ for $i \leq n$ for every $x \in U_{i}$ there is a canonical path connecting $x$ with $g^{-1} f(x)$. These paths define a fiberwise deformation $H: U_{i} \times[0,1] \rightarrow X^{\prime}$ of $U_{i}$ into $g^{-1} f\left(U_{i}\right) \subset X^{\prime}$ by the formula $H(x, t)=\phi_{i}^{x}(1-t)$.

\section{The main result}

We recall that the homotopical dimension of a space $X$, $\mathrm{hd}(X)$, is the minimal dimension of a CW-complex homotopy equivalent to $X$ [3].

Proposition 3.1 Let $p: E \rightarrow X$ be a fibration with $(n-1)$-connected fiber where $n=\operatorname{hd}(X)$. Then $p$ admits a section.

Proof Let $h: Y \rightarrow X$ be a homotopy equivalence with the homotopy inverse $g: X \rightarrow Y$ where $Y$ is a CW-complex of dimension $n$. Since the fiber of $p$ is $(n-1)$-connected, the map $h$ admits a lift $h^{\prime}: Y \rightarrow E$. Let $H$ be a homotopy connecting $h \circ g$ with $1_{X}$. By the homotopy lifting property there is a lift $H^{\prime}: X \times I \rightarrow E$ of $H$ with $\left.H\right|_{X \times\{0\}}=h^{\prime} \circ g$. Then the restriction $\left.H\right|_{X \times\{1\}}$ is a section.

We recall that $\lceil x\rceil$ denotes the smallest integer $n$ such that $x \leq n$.

Lemma 3.2 Suppose that a stratified locally trivial bundle $f: X \rightarrow Y$ with a fiber $(Z, A)$ is such that $Z$ is $r$-connected, $A$ is $(r-1)$-connected, $A$ is contractible in $Z$, and $Y$ is locally contractible. Then

$$
\text { cat }_{\mathrm{LS}} X \leq \operatorname{dim} Y+\left\lceil\frac{\operatorname{hd}(X)-r}{r+1}\right\rceil .
$$


Proof Let $\operatorname{dim} Y=m$ and $\operatorname{hd}(X)=n$.

By Lemma 2.3 the fiber $K$ of the fibration $\xi_{k}: E_{k} \rightarrow X$ is the join product $*^{k+1} F$ of $k+1$ copies of the fiber $F$ of the map $p_{A}: A_{Z} \rightarrow Z$. By Proposition 2.1, $F$ admits a fibration $\phi: F \rightarrow A$ with fibers homotopy equivalent to the loop space $\Omega Z$. Since the base $A$ and the fibers are $(r-1)$-connected, $F$ is $(r-1)$-connected. Thus, $K$ is $(k+(k+1) r-1)$-connected. By Proposition 3.1 there is a section $\sigma: X \rightarrow E_{k}$ to the fibration $\xi_{k}: E_{k} \rightarrow X$, whenever $k(r+1)+r \geq n$. Let $k$ be the smallest integer satisfying this condition. Thus, $k=\lceil(n-r) /(r+1)\rceil$.

By Lemma 2.3 a section $\sigma: X \rightarrow E_{k}$ defines a cover $\mathcal{U}=\left\{U_{0}, \ldots, U_{k}\right\}$ by sets fiberwise contractible to $X^{\prime}$ where $X^{\prime} \subset X$ is the first stratum. Let $\mathcal{U}_{m}=\left\{U_{0}, \ldots, U_{k+m}\right\}$ be an extension of $\mathcal{U}$ to a $(k+1)$-cover of $X$ from Corollary 1.5.

Let $\mathcal{O}$ be an open cover of $Y$ such that $f$ is trivial stratified bundle over each $O \in \mathcal{O}$. Let $\mathcal{C}$ be an open cover of $Y$ such that for every $C \in \mathcal{C}$ there is $O \in \mathcal{O}$ such that $C \subset O$ and $C$ is contractible in $O$. Such a cover exists since $Y$ is locally contractible. By Theorem 1.3 there are $m+k+1$ families of open sets $\mathcal{V}_{0}, \ldots, \mathcal{V}_{m+k}$ such that their union forms an $(m+1)$-cover of $Y$ refining $\mathcal{C}$. We define $V_{i}=\bigcup_{\alpha} V_{i}^{\alpha}$ to be the unions of all sets from $\mathcal{V}_{i}=\left\{V_{i}^{\alpha}\right\}$. Then $\mathcal{V}=\left\{V_{0}, \ldots, V_{m+k}\right\}$ is an open $(m+1)$-cover of $Y$ such that for every $i, V_{i}=\bigcup_{\alpha} V_{i}^{\alpha}$ is a disjoint union of open sets $V_{i}^{\alpha}$ contractible to a point in $O_{i}^{\alpha} \in \mathcal{O}$.

We show that for all $i \in\{0,1, \ldots, m+k\}$, the sets $W_{i}=f^{-1}\left(V_{i}\right) \cap U_{i}$ are contractible in $X$. Since

$$
W_{i}=\bigcup_{\alpha} f^{-1}\left(V_{i}^{\alpha}\right) \cap U_{i}
$$

is a disjoint union, it suffices to show that the sets $f^{-1}\left(V_{i}^{\alpha}\right) \cap U_{i}$ are contractible in $X$ for all $\alpha$. By Corollary 1.5 the set $U_{i}$ is fiberwise contractible into $X^{\prime}$ for $i \leq m+k$. Hence we can contract $f^{-1}\left(V_{i}^{\alpha}\right) \cap U_{i}$ to $f^{-1}\left(V_{i}^{\alpha}\right) \cap X^{\prime} \cong V_{i}^{\alpha} \times A$ in $X$. Then we apply a contraction to a point of $V_{i}^{\alpha}$ in $O_{i}^{\alpha}$ and $A$ in $F$ to obtain a contraction to a point of $f^{-1}\left(V_{i}^{\alpha}\right) \cap X^{\prime} \cong V_{i}^{\alpha} \times A$ in $f^{-1}\left(O_{i}^{\alpha}\right) \cong O_{i}^{\alpha} \times F$.

Next we show that $\left\{W_{i}\right\}_{i=0}^{m+k}$ is a cover of $X$. Since $\mathcal{V}$ is an $(m+1)-$ cover, by Proposition 1.2 every $y \in Y$ is covered by at least $k+1$ elements $V_{i_{0}}, \ldots, V_{i_{k}}$ of $\mathcal{V}$. Since $\mathcal{U}_{m}$ is a $(k+1)$-cover, $U_{i_{0}}, \ldots, U_{i_{k}}$ is a cover of $X$. Hence $W_{i_{0}}, \ldots, W_{i_{k}}$ covers $f^{-1}(y)$.

Theorem 3.3 For every $C W$-complex $X$ with the following inequality holds true:

$$
\operatorname{cat}_{\mathrm{LS}} X \leq \operatorname{cd}\left(\pi_{1}(X)\right)+\left\lceil\frac{\operatorname{hd}(X)-1}{2}\right\rceil .
$$


Proof Let $\pi=\pi_{1}(X)$ and let $\tilde{X}$ denote the universal cover of $X$. We consider Borel's construction:

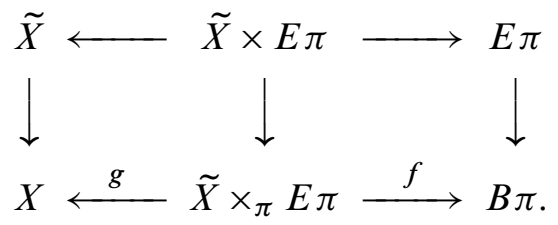

We refer for the properties of Borel's construction also known as the twisted product to [1]. Note that the 1 -skeleton $X^{(1)}$ of $X$ defines a $\pi$-equivariant stratification $\tilde{X}^{(1)} \subset \tilde{X}$ of the universal cover. This stratification allows us to treat $f$ as a stratified locally trivial bundle with the fiber $\left(\tilde{X}, \tilde{X}^{(1)}\right)$. We note that all conditions of Lemma 3.2 are satisfied for $r=1$. Therefore,

$$
\operatorname{cat}_{\mathrm{LS}}\left(\tilde{X} \times_{\pi} E \pi\right) \leq \operatorname{dim} B \pi+\left\lceil\frac{\operatorname{hd}\left(\tilde{X} \times_{\pi} E \pi\right)-1}{2}\right\rceil .
$$

Since $g$ is a fibration with homotopy trivial fiber, the space $\tilde{X} \times_{\pi} E \pi$ is homotopy equivalent to $X$. Thus, $\operatorname{cat}_{\mathrm{LS}}\left(\tilde{X} \times_{\pi} E \pi\right)=\operatorname{cat}_{\mathrm{LS}} X$ and hd $\left(\tilde{X} \times_{\pi} E \pi\right)=\operatorname{hd}(X)$. In view of the results of Eilenberg and Ganea [7] (see also Brown [2]) we may assume that $\operatorname{dim} B \pi=\operatorname{cd}(\pi)$ if $\operatorname{cd}(\pi)>2$. The case when $\operatorname{cd}(\pi) \leq 2$ is treated in [5].

Acknowledgements This work was partially supported by NSF grant DMS-0904278. I would like to thank the anonymous referee for helpful comments on an earlier draft.

\section{References}

[1] G E Bredon, Introduction to compact transformation groups, 46, Academic Press, New York (1972) MR0413144

[2] K S Brown, Cohomology of groups, Graduate Texts in Math. 87, Springer, New York (1982) MR672956

[3] O Cornea, G Lupton, J Oprea, D Tanré, Lusternik-Schnirelmann category, Math. Surveys and Monogr. 103, Amer. Math. Soc. (2003) MR1990857

[4] A Dold, Partitions of unity in the theory of fibrations, Ann. of Math. (2) 78 (1963) 223-255 MR0155330

[5] A N Dranishnikov, On the Lusternik-Schnirelmann category of spaces with 2dimensional fundamental group, Proc. Amer. Math. Soc. 137 (2009) 1489-1497 MR2465675

[6] A N Dranishnikov, MG Katz, Y B Rudyak, Small values of the LusternikSchnirelmann category for manifolds, Geom. Topol. 12 (2008) 1711-1727 MR2421138 
[7] S Eilenberg, T Ganea, On the Lusternik-Schnirelmann category of abstract groups, Ann. of Math. (2) 65 (1957) 517-518 MR0085510

[8] DP Grossman, An estimation of the category of Lusternik-Schnirelmann, C. R. (Doklady) Acad. Sci. URSS (N.S.) 54 (1946) 109-112 MR0018820

[9] W Hurewicz, On the concept of fiber space, Proc. Nat. Acad. Sci. USA 41 (1955) 956-961 MR0073987

[10] PA Ostrand, Dimension of metric spaces and Hilbert's problem 13, Bull. Amer. Math. Soc. 71 (1965) 619-622 MR0177391

[11] E H Spanier, Algebraic topology, McGraw-Hill, New York (1966) MR0210112

[12] J Stallings, Groups of dimension 1 are locally free, Bull. Amer. Math. Soc. 74 (1968) 361-364 MR0223439

[13] J Strom, Lusternik-Schnirelmann category of spaces with free fundamental group, Algebr. Geom. Topol. 7 (2007) 1805-1808 MR2366179

[14] A S Švarc, The genus of a fibered space, Trudy Moskov. Mat. Obšč. 10 (1961) 217-272 MR0154284

[15] R G Swan, Groups of cohomological dimension one, J. Algebra 12 (1969) 585-610 MR0240177

[16] G W Whitehead, The homology suspension, from: "Colloque de topologie algébrique, Louvain, 1956”, Georges Thone, Liège (1957) 89-95 MR0094794

Department of Mathematics, University of Florida PO Box 118105, 358 Little Hall, Gainesville, FL 32611-8105

dranish@math.ufl.edu

Received: 23 September 2009 Revised: 16 February 2010 